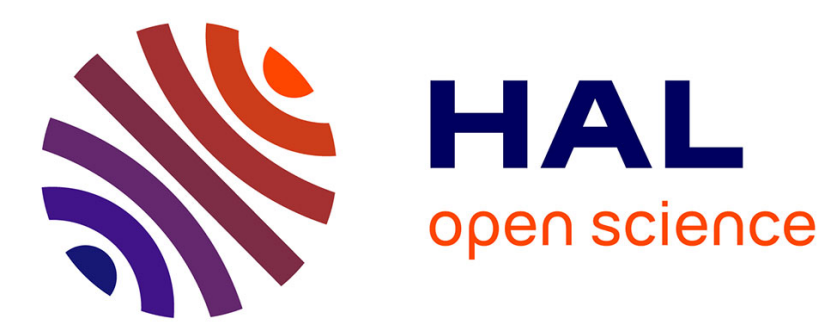

\title{
Numerical assessment of 3-D macrodispersion in heterogeneous porous media
}

\author{
Anthony Beaudoin, Jean-Raynald de Dreuzy
}

\section{To cite this version:}

Anthony Beaudoin, Jean-Raynald de Dreuzy. Numerical assessment of 3-D macrodispersion in heterogeneous porous media. Water Resources Research, 2013, 49 (5), pp.2489-2496. 10.1002/wrcr.20206 . insu-00907370

\section{HAL Id: insu-00907370 \\ https://hal-insu.archives-ouvertes.fr/insu-00907370}

Submitted on 21 May 2014

HAL is a multi-disciplinary open access archive for the deposit and dissemination of scientific research documents, whether they are published or not. The documents may come from teaching and research institutions in France or abroad, or from public or private research centers.
L'archive ouverte pluridisciplinaire HAL, est destinée au dépôt et à la diffusion de documents scientifiques de niveau recherche, publiés ou non, émanant des établissements d'enseignement et de recherche français ou étrangers, des laboratoires publics ou privés. 


\title{
Numerical assessment of 3-D macrodispersion in heterogeneous porous media
}

\author{
A. Beaudoin ${ }^{1}$ and J.-R. de Dreuzy ${ }^{2,3}$ \\ Received 1 August 2012; revised 31 January 2013; accepted 20 March 2013; published 22 May 2013.
}

[1] Hydrodynamic dispersion is a key controlling factor of solute transport in heterogeneous porous media that critically depends on dimensionality. The transverse macrodispersion (asymptotic dispersion transverse to the mean velocity direction) is known to vanish only in 2-D and not in 3-D. Using classical Gaussian correlated permeability fields with a lognormal distribution of variance $\sigma_{Y}^{2}$, we determine numerically the longitudinal and transverse dispersivities as functions of heterogeneity and dimensionality. We show that the transverse macrodispersion steeply increases with $\sigma_{Y}^{2}$ underlying the essential role of flow lines braiding, a mechanism specific to 3 -D systems that we qualitatively characterize by the increasing expansion of the flow lines transversally to the flow direction. The transverse macrodispersion remains however at least two orders of magnitude smaller than the longitudinal macrodispersion, which increases even more steeply with $\sigma_{Y}^{2}$. At moderate to high levels of heterogeneity, the transverse dispersion also converges much faster to its asymptotic regime than do the longitudinal dispersion. Braiding cannot be thus taken as the sole mechanism responsible for the high longitudinal macrodispersions. It could be either supplemented or superseded by stronger velocity correlations in 3-D than in 2-D. This assumption is supported by the much larger longitudinal macrodispersions obtained in 3-D than in 2-D, up to a factor of 5 for $\sigma_{Y}^{2}=6.25$.

Citation: Beaudoin, A., and J.-R. de Dreuzy (2013), Numerical assessment of 3-D macrodispersion in heterogeneous porous media, Water Resour. Res., 49, 2489-2496, doi:10.1002/wrcr.20206.

\section{Introduction}

[2] Hydrodynamic dispersion is a major component of solute transport in geological media controlling the relative solute distribution [Bear, 1973; Saffman, 1959] as well as the mixing-induced chemical reactivity [Chiogna et al., 2011; Cirpka and Kitanidis, 2000; Cirpka et al., 2011; de Dreuzy et al., 2012b; de Simoni et al., 2005; Jha et al., 2011; Le Borgne et al., 2010, 2011]. It results from the variability and the correlation of velocity fields over evolving scales from the pore scale to the aquifer scale [e.g., Dagan, 1989; Gelhar, 1993; Lallemand-Barres and Peaudecerf, 1978; Neuman et al., 1987; Rubin, 1990; Taylor, 1953]. Hydrodynamic dispersion appears to be a Fickian process where the diffusion coefficient is replaced by an equivalent dispersion coefficient, which increases with time as the solute plume samples the heterogeneity field.

[3] The relation of geological heterogeneity to dispersion processes has been extensively analyzed in correlated

\footnotetext{
${ }^{1}$ Institute P' (UPR CNRS 3346), University of Poitiers, Poitiers, France.

${ }^{2}$ Institute of Environmental Analysis and Water Studies, CSIC, Barcelona, Spain.

${ }^{3}$ Géosciences Rennes (UMR CNRS 6118), Campus de Beaulieu, Université de Rennes 1, Rennes, France.

Corresponding author: A. Beaudoin, Institute P' (UPR CNRS 3346), University of Poitiers, 86962 Futuroscope Chassemeuil, Poitiers F-35042,

France. (Anthony.Beaudoin@univ-poitiers.fr)
}

multi-Gaussian log-permeability fields taken as an idealized model both simple enough to lead to general conclusions and complex enough to disclose some of the relationships between heterogeneity and dispersion [e.g., Dagan, 1984; Delhomme, 1979; Freeze, 1975; Gelhar and Axness, 1983]. The logarithm of permeability $Y(\mathbf{x})=\ln (K(\mathbf{x}))$ follows a normal distribution of variance $\sigma_{Y}^{2}$ following a simple correlation pattern. Here we will be considering a simple isotropic Gaussian correlation function such as:

$$
\left\langle Y^{\prime}(\mathbf{x}) Y^{\prime}\left(\mathbf{x}^{\prime}\right)\right\rangle=\sigma_{Y}^{2} \exp \left(-\left(\frac{\left|\mathbf{x}-\mathbf{x}^{\prime}\right|}{\lambda}\right)^{2}\right),
$$

where $Y^{\prime}(\mathbf{x})=Y(\mathbf{x})-\langle Y\rangle,\langle\rangle$ marks the spatial average and $\lambda$ is the correlation length. Whenever heterogeneities are bounded and their correlation limited, dispersion reaches an asymptotic regime characterized by an asymptotic dispersion coefficient also called macrodispersion. Perturbation expansions of the flow and advection-diffusion equations have shown that macrodispersion is strongly anisotropic [Dagan, 1984, 1989; Dentz et al., 2000; Gelhar and Axness, 1983; Hsu, 2003; Neuman and Zhang, 1990; Rubin, 1990]. In the direction longitudinal to the mean velocity, the macrodispersion $D_{\mathrm{LA}}$ is proportional to the correlation length times the lognormal permeability variance:

$$
D_{\mathrm{LA}}=\frac{\sqrt{\pi}}{2}\langle v\rangle \lambda \sigma_{Y}^{2},
$$


with $\langle v\rangle$ the mean velocity. The factor $\frac{\sqrt{\pi}}{2}$ comes from the Gaussian-type of the correlation and the chosen definition of the correlation function of equation (1). In the transverse directions, the macrodispersion $D_{T A}$ is zero in the absence of local diffusion, i.e., when the local Peclet number is infinite or equivalently when velocity heterogeneities are the sole source of dispersion:

$$
D_{\mathrm{TA}}=0
$$

[4] These analytical estimates have been derived both in 2-D and in 3-D for small enough permeability heterogeneities $\left(\sigma_{Y}^{2}<1\right)$. They have been confirmed in 2-D by numerous numerical studies [Dentz et al., 2002; Rubin, 1990; Schwarze et al., 2001] and have even been found to be robust for values of $\sigma_{Y}^{2}$ as high as 1.6 [Bellin et al., 1992] and even higher for spherical inclusion systems [Jankovic et al., 2003]. At higher heterogeneity levels $\left(1<\sigma_{Y}^{2} \leq 9\right)$, the absence of transverse dispersion in 2-D has been confirmed both by homogenization theory and numerical simulations [Attinger et al., 2004; de Dreuzy et al., 2007; Lunati et al., 2002]. The basic argument is that, in 2-D without local diffusion, the solute plume is constrained within a given flow tube of finite and stable transverse dimension that strictly limits the dispersion. On the contrary for the longitudinal macrodispersion, numerical simulations have shown that it can be up to three times larger than the analytical estimate and that it scales with the square of $\sigma_{Y}^{2}$ for $\sigma_{Y}^{2} \geq 2$ [de Dreuzy et al., 2007]. The strong increase of the macrodispersion solely comes from the structure of the flow lines because of the absence of local diffusive and dispersive processes. It is linked to the wider velocity distributions and to the higher-order velocity correlations like the dependence of the velocity correlation on the velocity magnitude [Englert et al., 2006; Le Borgne et al., 2007].

[5] In 3-D, full transport simulations on the linearized flow equation show that the transverse macrodispersion $D_{T A}$ is significantly nonzero [Dentz et al., 2002; Schwarze et al., 2001], which proves to be consistent with predictions based on Corrsin's conjecture [Neuman and Zhang, 1990], and coarse-graining arguments [Attinger et al., 2004]. For spherical inclusion systems, the transverse macrodispersion is also nonvanishing but it remains small. It becomes critically larger for elongated ellipsoids [Jankovic et al., 2003, 2009]. If it has been well established that the transverse macrodispersion is nonzero, open questions remain on its magnitude as well as on the magnitude of the longitudinal macrodispersion and on their behavior for higher heterogeneity levels $\left(\sigma_{Y}^{2}>1\right)$. We address these issues with 3-D extensive simulations of the full flow and transport equations that go far beyond the previous attempts [Chin and Wang, 1992; Tompson and Gelhar, 1990; Trefry et al., 2003; Zhang and Seo, 2004].

\section{Model and Methods}

[6] The permeability field (1) is generated within a rectangular parallelepiped of dimensions $L_{\mathrm{L}} \times L_{\mathrm{T} 1} \times L_{\mathrm{T} 2}$ at the same resolution $\Delta$ in all directions using a spectral method through the fftw library [Frigo and Johnson, 2005; Gutjahr, 1989]. The indices " $L "$, " $T 1$ " and " $T 2$ " stand for the three orthogonal directions of the parallelepiped and symbolize the direction " $L$ " longitudinal to the main flow direction (defined just after) and the two directions " $T 1$ " and " $T 2$ " orthogonal to it. The flow equation $\nabla(K \nabla h)=0$ is solved with fixed heads $h_{+}$and $h$ - on the faces orthogonal to the longitudinal direction $L$. Periodic boundary conditions on the other transverse faces minimize the perturbation of the flow field generally reported under no-flow boundary conditions [Englert et al., 2006; Salandin and Fiorotto, 1998]. The flow equation is discretized by a finite-volume scheme with a harmonic composition rule of the permeability between adjacent mesh cells [Chavent and Roberts, 1991; Roberts and Thomas, 1991]. The resulting linear system is solved with the parallel algebraic multigrid method of HYPRE [Falgout et al., 2005]. Velocity $\boldsymbol{v}$ at the grid center is derived from the hydraulic head $h$ by Darcy's law $\mathbf{v}=-K / \theta \nabla h$. Porosity $\theta$ is chosen uniform and the mean flow velocity $\langle\boldsymbol{v}\rangle$ is taken parallel to the direction $L$. Its norm $\|<\boldsymbol{v}>\|$ is equal to $\left(K_{e q} / \theta\right)\left|h_{+}-h_{-}\right| / L_{L}$ with $K_{\text {eq }}$ the equivalent permeability [Matheron, 1967]. Transport is restricted to the sole advection process and is simulated by a first-order explicit particle tracking method [Kinzelbach, 1988; van Kampen, 1981]. Between $t$ and $t+\mathrm{d} t$, a particle moves from the position $\boldsymbol{x}(t)$ to the position $\boldsymbol{x}(t+\mathrm{d} t)$ according to $\mathbf{x}(t+\mathrm{d} t)=\mathbf{x}(t)+\mathbf{v}[\mathbf{x}(t)] \mathrm{d} t$ where the velocity $\boldsymbol{v}$ in each point is obtained with a linear interpolation in each direction from its grid values [Pollock, 1988]. The time step $\mathrm{d} t$ is adapted along the particle trajectory to the magnitude of the local velocity so that the particle takes on average 10 steps within each grid cell [Wen and Gomez-Hernandez, 1996]. Particles are injected on a large plane of size 0.8 $L_{T 1} \times 0.8 L_{T 2}$ orthogonal to the direction $L$ located at least at five correlation lengths downstream from the side of the system to avoid border effects on the velocity statistics. Particles are injected proportionally to the flow through the plane to speed up the convergence to the asymptotic regime. Effective dispersivities derived from the dispersion coefficients divided by the mean velocity $\|\langle\boldsymbol{v}\rangle\|$ and the correlation length $\lambda$ of the isotropic Gaussian correlation function (equation (1)) are determined from $N_{R}$ realizations of the permeability field with $N_{p}$ particles for each realization according to:

$$
\alpha_{k}(t)=\frac{1}{2 \lambda\|\langle\mathbf{v}\rangle\|} \frac{1}{N_{R}} \sum_{i=1}^{N_{R}} \frac{\mathrm{d}}{\mathrm{d} t}\left[\frac{1}{N_{p}} \sum_{j=1}^{N_{p}} x_{k}^{j, i}(t)^{2}-\left(\frac{1}{N_{p}} \sum_{j=1}^{N_{p}} x_{k}^{j, i}(t)\right)^{2}\right]
$$

where $x_{k}^{j, i}$ is the position of the particle $j$ in the simulation $i$ in the direction $k$ at time $t$. For consistency we have normalized the time $t$ by $\lambda /\|\langle\mathbf{v}\rangle\|$ and kept the same notation $t$ for simplicity. As we have systematically checked that $\alpha_{\mathrm{T} 1}=\alpha_{\mathrm{T} 2}$, we report results only for one of them that we generically denote $\alpha_{\mathrm{T}}$. To reach large enough domain sizes and number of simulations, all numerical methods have been implemented in parallel [Beaudoin et al., 2005, 2007, 2010; Erhel et al., 2008, 2009]. More details on the methods and their implementation can be found in similar previous studies on 2-D macrodispersion [Beaudoin et al., 2006; de Dreuzy et al., 2007].

[7] We have extensively studied the convergence as a function of the numerical parameters of the model 

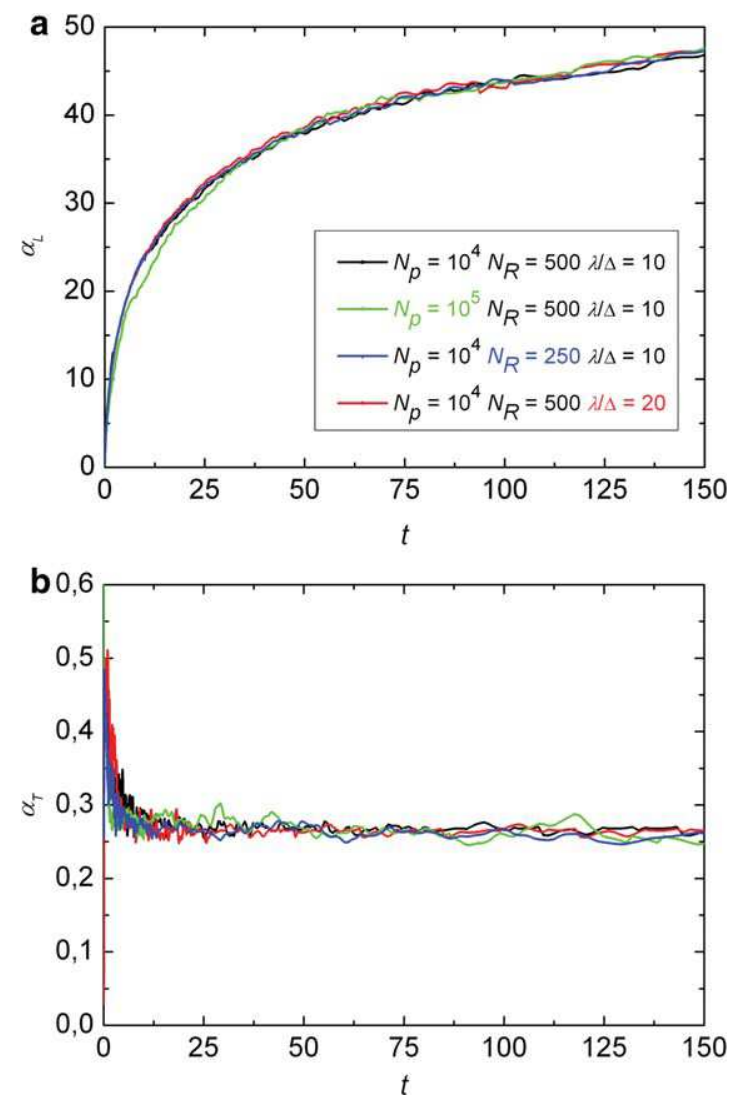

Figure 1. Convergence of the time-dependent dimensionless (a) longitudinal and (b) transverse effective dispersivities, $\alpha_{L}$ and $\alpha_{T}$, with $\lambda / \Delta, N_{p}$ and $N_{R}$, for $\sigma_{y}{ }^{2}=6.25$ and $L_{T} / \lambda=25.6$.

including the number $N_{p}$ of particles, the number $N_{R}$ of realizations and the resolution $\lambda / \Delta$ of the permeability field. We synthesize the convergence analysis in Figure 1 which shows the time-dependent dimensionless dispersivities in both the longitudinal and transverse directions for different parameter sets at $\sigma_{Y}^{2}=6.25$. The black line stands for the reference simulation with $N_{p}=10^{4}, N_{R}=500$ and $\lambda / \Delta=10$. Each of the other curves illustrates the modification of dispersivities when altering one of those reference parameters, all other parameters kept constant. The number of particles $N_{p}$ is increased by a factor 10 (green curve). The number of realizations is decreased from 500 to 250 (blue curve). The resolution $\lambda / \Delta$ increases from 10 to 20 (red curve). Overall, the different cases show limited variations with time without any significant and systematic tendency. The asymptotic dispersion coefficients $\alpha_{L A M}$ and $\alpha_{T A M}$ determined for all cases as the average of the time-dependent dispersivities over the last fourth of the presented time span do not vary more than $2 \%$ in the longitudinal direction and $4 \%$ in the transverse direction. Smaller $\sigma_{Y}^{2}$ cases lead to similar conclusions. We have thus kept the set of the reference parameters for all presented simulations: $N_{p}=10^{4}, N_{R}=500, \lambda / \Delta=10$. Such values lead to 25 particles per correlation square within the injection window (square of $\lambda^{2}$ ) and to at least $210^{5}$ sample unit squares over the 500 realizations. Additional sensitivity tests on the size of injection window have shown that its transverse dimension of around $(20 \lambda)^{2}=400 \lambda^{2}$ is more than enough to simulate ergodic initial conditions and to provide an exhausting sampling of the velocity field.

\section{Results}

[8] We study the temporal development of the longitudinal and transverse dispersivities in the first subsection in order to determine relevant ways to estimate the macrodispersivities presented in the second subsection.

\subsection{Temporal Development of Dispersivities}

[9] Figure 2 displays the temporal evolution of the dimensionless effective dispersivities as computed by equation (4). Particles are injected proportionally to the local flows in most of the transverse section. These conditions ensure the consistency of the velocity field sampling and minimize the convergence time to the asymptotic regime. The time necessary to reach the asymptotic regime is around the advection time $(\lambda \|<\boldsymbol{v}\rangle \|)$ at low levels of heterogeneity for the longitudinal dispersivity (Figure 2a, $\left.\sigma_{Y}^{2} \leq 1\right)$ and for the transverse dispersivity at all levels of heterogeneity (Figure 2b). At 10 times the advection time (corresponding on the Figure 2 to 10 because of the normalization), the transverse macrodispersivity as well as the
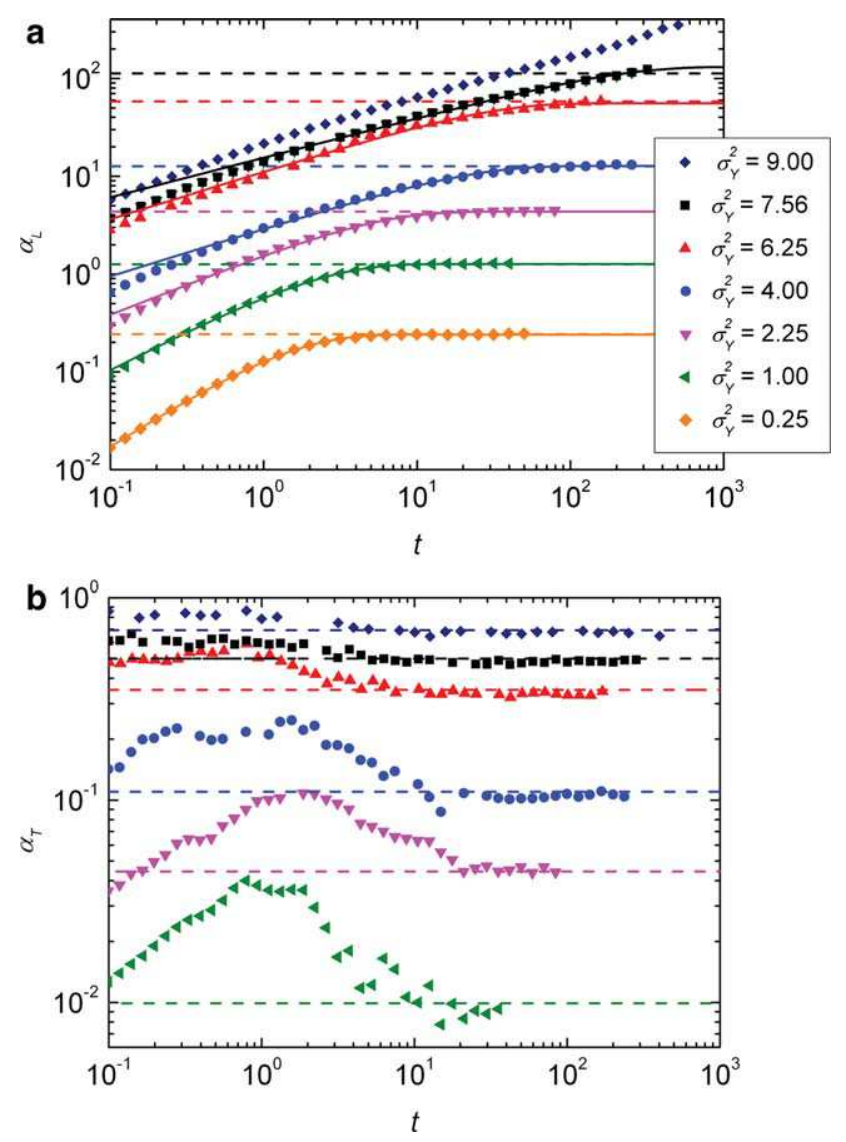

Figure 2. Time evolution of the dimensionless (a) longitudinal and (b) transverse effective dispersivities $\alpha_{L}(t)$ and $\alpha_{T}(t)$ as defined by equation (4) for various values of $\sigma_{Y}^{2}$. Full and dashed lines stand respectively for $\alpha_{L A M}$ (equation (5)) and for the fit $\alpha_{L T}(t)$ (equation (6)). 
longitudinal macrodispersivity for $\sigma_{Y}^{2} \leq 1$ (green and orange curves) have converged to their asymptotic values. In these cases, a relatively small system size $L_{L}$ of $102.4 \lambda$ is enough to reach the convergence. The macrodispersivity can be directly estimated as the average of the effective dispersivity once the asymptotic regime has settled. Practically, we take the average of $\alpha_{k}(t)$ over the last fourth of the simulation time range extending from the injection time $(t=0)$ to the first breakthrough time $t_{b}$ :

$$
\alpha_{k A M}=\frac{1}{0.25 t_{b}} \int_{0.75 t_{b}}^{t_{b}} \alpha_{k A M}(t) d t
$$

where $\alpha_{k A M}$ is the macrodispersivity in direction $k$. The indices " $A$ " and " $M$ " stand respectively for the words asymptotic and mean (late-time average). The first breakthrough time $t_{b}$ corresponds to the time at which the first particle steps out of the simulation domain. Since the evaluation of plume statistics would be biased at later times, we limit the time range to $t_{b}$. The average over a wide interval of a fourth of $t_{b}$ filters out the small temporal variations still remaining after averaging over 500 realizations.

[10] The definition (5) of the asymptotic macrodispersivity can be used for the transverse macrodispersivity at all levels of heterogeneity and for the longitudinal macrodispersivity at low to intermediary levels of heterogeneities $\left(\sigma_{Y}^{2} \leq 4\right)$. However, for the longitudinal macrodispersivity at the highest levels of heterogeneities $\left(\sigma_{Y}^{2}>6.25\right)$, the convergence is much slower to establish and, at best, just reached in the available time range or not yet reached (Figure 2a). $\alpha_{L A M}$ is a lower estimate of the effective asymptotic dispersivity. The longitudinal system size has been set to the maximum that we could reach $\left(L_{L} / \lambda=1638.4\right)$. We define an alternative characterization of the longitudinal macrodispersivity by extending the rate of convergence of the preasymptotic regime to convergence through an empirical well chosen fit. Longitudinal effective dispersivity first increases linearly with time $(t<1)$, than as a power law and eventually converges exponentially to its asymptotic value (Figure 2a). For $t>1$, the temporal development of effective dispersivity can thus be heuristically described by:

$$
f_{L}(t)=\alpha_{L A F}\left(1-\exp \left(-t / t_{L F}\right)\right)^{a_{L F}}
$$

with $\alpha_{L A F}$ the estimated longitudinal macrodispersivity, $t_{L F}$ a characteristic convergence time to the longitudinal asymptotic regime and $a_{L F}$ the characteristic power-law exponent of the initial increase. The index " $F$ " stands for Fitting. The best fit of $\alpha_{L}(t)$ by $f_{L}(t)$ displayed on Figure 2a by the lines shows the relevance of equation (6) to the longitudinal effective dispersivity at all heterogeneity levels. The initial power-law increase characterized by the exponent $a_{L F}$ strongly depends on the heterogeneity level (Table 1). At low levels of heterogeneities, it is linear consistently with available predictions (equation (1)) while, at higher levels of heterogeneities, the rate of increase slows down critically to scale as $t^{0.4}$ at $\sigma_{Y}^{2}=7.56$. Note that this type of slow powerlaw increase is specific to the 3-D case, as the 2-D longitudinal effective dispersivity follows more a linear and then exponential rate of convergence at high heterogeneity levels [de Dreuzy et al., 2007]. The characteristic time to reach the
Table 1. Fitting Parameters Obtained With Equation (6) for the Longitudinal Effective Dispersivity

\begin{tabular}{lccc}
\hline$\sigma_{Y}^{2}$ & $\alpha_{L A F}$ & $t_{L F}$ & $a_{L F}$ \\
\hline 0.25 & 0.24 & 1.35 & 1 \\
1 & 1.27 & 2.21 & 0.80 \\
2.25 & 4.35 & 4.83 & 0.63 \\
4 & 12.7 & 20.5 & 0.49 \\
6.52 & 58.6 & 41.9 & 0.40 \\
7.56 & 131 & 208 & 0.39 \\
\hline
\end{tabular}

asymptotic regime $t_{L F}$ also sharply increases with $\sigma_{Y}^{2}$. It increases exponentially over 2 orders of magnitude from $\sigma_{Y}^{2}=1$ to 7.56 (Figure 3). The asymptotic regime has never been approached for $\sigma_{Y}^{2}=9$ in the longitudinal direction within the available simulation time (Figure 2a). We conclude that the transverse effective dispersivity converges much faster than the longitudinal effective dispersivity to the asymptotic regime at high heterogeneity levels and that heterogeneity slows down critically the convergence rate to the longitudinal macrodispersivity. Whatever the case, the previous analysis gives ways to estimate the macrodispersivities. In the transverse direction, macrodispersivity can be estimated by the late-time averaging of equation (5). In the longitudinal direction, both the late-time estimation and the fitting methods of equations (5) and (6) leading to $\alpha_{L A M}$ and $\alpha_{L A F}$ give nearly equal predictions for $\sigma_{Y}^{2} \leq 4$ while $\alpha_{L A F}$ becomes sensibly larger than $\alpha_{L A M}$ above.

\subsection{Macrodispersivities}

[11] At low to moderate levels of heterogeneities $\left(\sigma_{Y}^{2} \leq 2.25\right)$, the values of macrodispersivities $\alpha_{L A M}$ and $\alpha_{\text {TAM }}$ obtained from the late-time averaging (equation (5)) and $\alpha_{L A F}$ obtained from equation (6) are compared to those obtained fully analytically by perturbative analysis [Gelhar and Axness, 1983] and half analytically by full transport simulations on a first-order approximation of the velocity field [Dentz et al., 2002; Schwarze et al., 2001]. At low level of heterogeneities, the numerically obtained macrodispersivities are closer to those of Gelhar and Axness. [1983] in the longitudinal direction and to those of Dentz

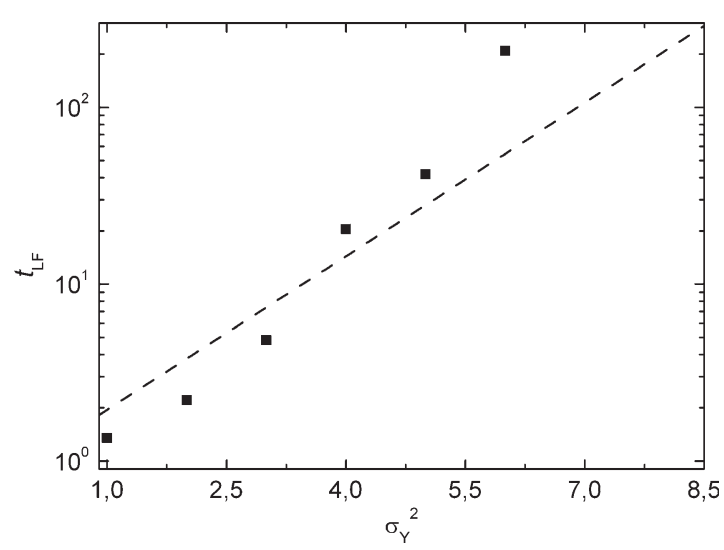

Figure 3. Characteristic convergence time $t_{L F}$ as defined by equation (6) to the asymptotic regime in the longitudinal direction as a function of $\sigma_{Y}^{2}$. The dashed line shows for comparison a close exponential function. 
Table 2. Longitudinal and Transverse Macrodispersivities Obtained From the Numerical Simulations Obtained by the Late-Time Averaging Method of Equation (6) (Column "This Study") Compared to the Perturbation Estimates [Gelhar and Axness, 1983] (Column "Gelhar") and to the Estimates Obtained on Synthetic Velocity Fields Derived From First-Order Approximation by Dentz et al. [2002] (Column "Dentz") and Schwarze et al. [2001] (Column "Schwarze")

\begin{tabular}{|c|c|c|c|c|c|c|c|c|}
\hline \multirow[b]{2}{*}{$\sigma_{Y}^{2}$} & \multicolumn{4}{|c|}{$\alpha_{L A}$} & \multicolumn{4}{|c|}{$\alpha_{T A}$} \\
\hline & Gelhar & Dentz & Schwarze & This study & Gelhar & Dentz & Schwarze & This study \\
\hline 0.25 & 0.22 & 0.28 & & 0.26 & 0 & 0 & & $<10-^{3}$ \\
\hline 1 & 0.89 & 1.34 & 1.24 & 1.27 & 0 & 0.007 & 0.009 & 0.01 \\
\hline 2.25 & 1.99 & 2.33 & & 4.35 & 0 & 0.028 & & 0.044 \\
\hline
\end{tabular}

et al. [2002] and Schwarze et al. [2001] in the transverse direction (see Table 2). In the longitudinal direction for $\sigma_{Y}^{2}>1$, divergence from the analytical approximations increases faster in 3-D than in 2-D.

[12] At moderate to high levels of heterogeneities $\left(\sigma_{Y}^{2} \geq 1\right)$, the values of macrodispersivities $\alpha_{L A F}, \alpha_{L A M}$ and $\alpha_{T A M}$ estimated from the numerical simulations are displayed on Figure 4. The transverse macrodispersivity is significantly nonzero as previously demonstrated [Attinger et al., 2004; Jankovic et al., 2009; Lunati et al., 2002] and strongly increases with $\sigma_{Y}^{2}$ (Figure $4 \mathrm{~b}$ ). More precisely it increases quadratically in $\sigma_{Y}^{2}$ for $\sigma_{Y}^{2} \geq 1$ (dashed line compared to black squares):

$$
\alpha_{T A M} \approx \alpha_{T A M}(1) \sigma_{Y}^{4}
$$

[13] The longitudinal macrodispersivity is much larger than the linear prediction of Gelhar and Axness [1983] and than its 2-D equivalent recomputed here using exactly the same assumptions as in 3-D and the late-time averaging method of equation (5). The strong difference to the full perturbative solution is expected as it was already the case in 2-D. The large differences between the 2-D and the 3-D cases show that the Euclidean dimension does not only impact the transverse macrodispersivity but also the longitudinal one. The 3-D longitudinal macrodispersivity is twice larger than the 2-D one for $\sigma_{Y}^{2}=2.25$ and 5 times larger for $\sigma_{Y}^{2}=6.25$. More globally, the 2-D and 3-D longitudinal macrodispersivities display different dependencies with $\sigma_{Y}^{2}$. In 2-D, the longitudinal macrodispersity increases first linearly and then quadratically with $\sigma_{Y}^{2}$ at high heterogeneities (blue dashed line and the blue squares) as also suggested on the basis of analytical approximations [Dagan, 1988; Gelhar, 1993]:

$$
\alpha_{L A M}(2 D) \approx 0.84 \sigma_{Y}^{2}+0.17 \sigma_{Y}^{4}
$$

[14] The coefficients in front of the linear and quadratic terms are only slightly different from those obtained with an exponentially correlated permeability field [de Dreuzy et al., 2007]. In 3-D, $\alpha_{L A F}$ and $\alpha_{L A M}$ are close together and increase rather exponentially than quadratically with $\sigma_{Y}^{2}$ for $\sigma_{Y}^{2} \geq 2.25$ (Figure $4 \mathrm{a}$, black dashed line compared to the black stars):

$$
\alpha_{L A M} \approx \exp \left(\sigma_{Y}^{2} / 1.55\right)
$$

[15] We underline that $\alpha_{L A F}$ and $\alpha_{L A M}$ are first estimates of the asymptotic longitudinal dispersivity that may be slightly modified by larger simulations. However, their exponential increase at moderate to high level of heterogeneities will remain relevant especially as $\alpha_{L A M}$ is an underestimate of the effective value for $\sigma_{Y}^{2} \geq 6.25$.

[16] While the longitudinal and transverse macrodispersivities display very different types of variations, their rate $\alpha_{L A F} / \alpha_{T A M}$ is less variable between 100 and 200, close to the value of 250 obtained for spherical inclusions at $\sigma_{Y}^{2}=4$ [Jankovic et al., 2003]. It remains very high showing that the longitudinal macrodispersivity is at least 2 orders of magnitude larger than the transverse macrodispersivity.
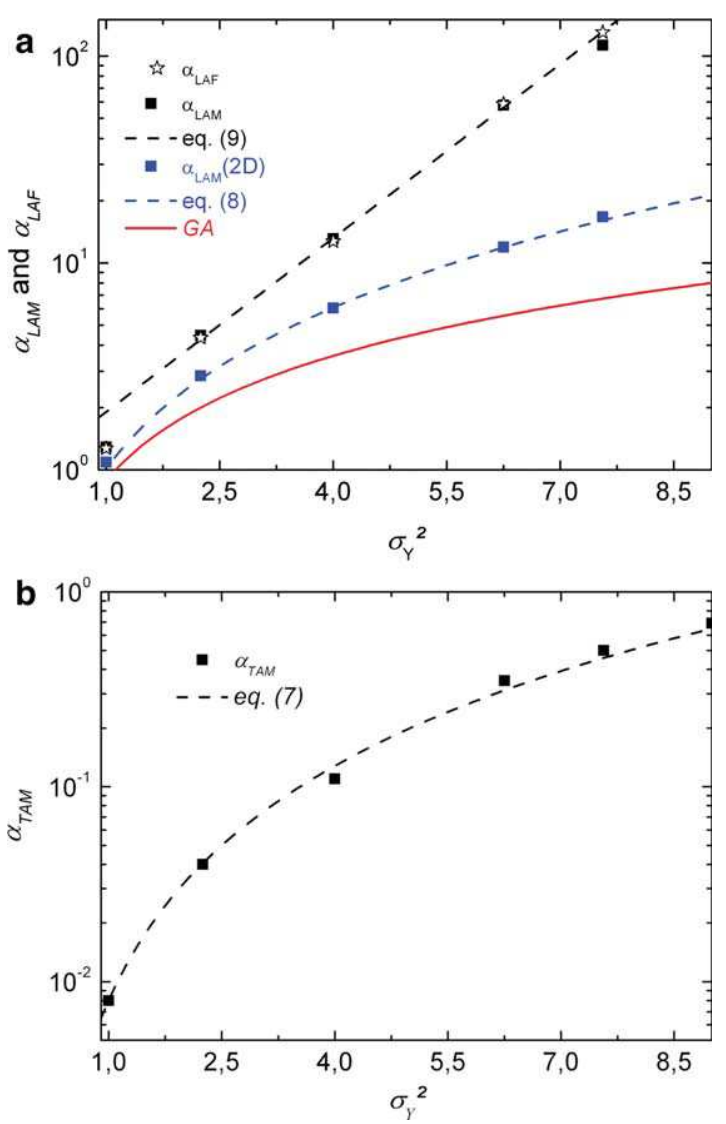

Figure 4. (a) Longitudinal and (b) transverse macrodispersivities estimated by fitting the time-dependent effective dispersivities $\left(\alpha_{L A F}\right)$ and by their long-time averaging $\left(\alpha_{L A M}\right.$ and $\left.\alpha_{T A M}\right)$ as functions of $\sigma_{Y}^{2}$. The longitudinal macrodispersivity (Figure $4 \mathrm{a}$ ) is compared to the values obtained numerically for equivalent 2-D systems (blue squares) and to the perturbative approximation (red line) [Gelhar and Axness, 1983]. 


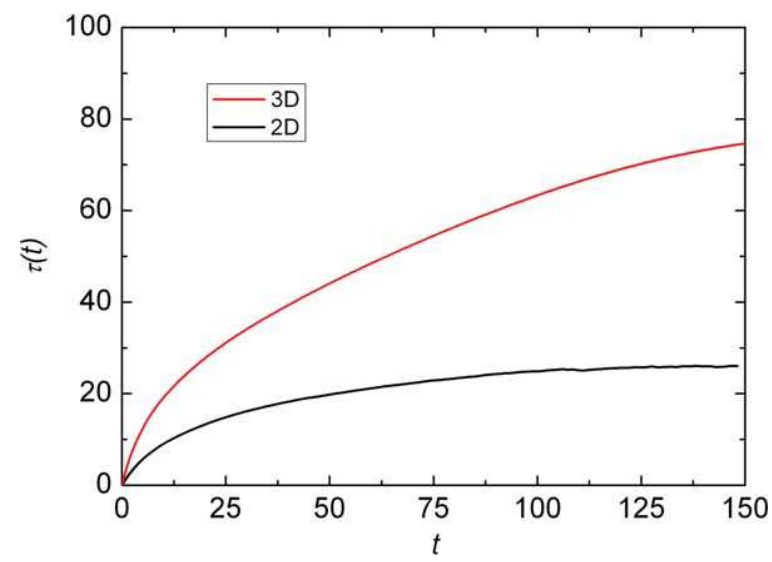

Figure 5. Comparison of the time-dependent transverse tortuosity parameter $\tau(t)$ in 2-D (black line) and 3-D (red line) as defined by equation (9) for $\sigma_{Y}^{2}=6.25, N_{p}=10^{4}, N_{R}$ $=500, \lambda \Delta=10$ and $L_{T} / \lambda=25.6$.

[17] We conclude that the longitudinal macrodispersivity increases exponentially with $\sigma_{Y}^{2}$ at high levels of heterogeneities $\left(\sigma_{Y}^{2} \geq 2.25\right)$. The increase is much stronger than both the linear increase predicted by perturbative analysis and the quadratic increase characteristic of its 2-D counterpart. The transverse macrodispersivity is significantly positive (nonzero) and increases quadratically with $\sigma_{Y}^{2}$. The longitudinal macrodispersivity remains always 2 orders of magnitude larger than the transverse macrodispersivity.

\section{Discussion and Conclusion}

[18] Nonvanishing 3-D transverse macrodispersivities has been explained in terms of the flow lines structure. In 3-D, flow lines can alternatively converge and diverge without intersecting [Attinger et al., 2004]. This mechanism already qualified as twiggling and intertwinning is enhanced by permeability structures that deflect the flow lines from the main flow direction as do for example elongated ellipsoidal inclusions [Jankovic et al., 2009]. For the case of highly heterogeneous media, deflecting comes from the low-velocity regions and induces a braiding of the channels (high-velocity zones). These mechanisms are negligible at low levels of heterogeneities $\left(\sigma_{Y}^{2} \leq 1\right)$ but are sharply enhanced at higher levels as demonstrated by the quadratic increase of the transverse macrodispersivity with $\sigma_{Y}^{2}$ for $\sigma_{Y}^{2} \geq 2.25$ (equation (7)).

[19] To get some more insights on the structure of the flow lines, we characterize the structure of the flow lines by a transverse dimensionless tortuosity parameter $\tau$ that evaluates their expansion transversally to the main flow direction:

$\tau(t)=\frac{1}{\|<v>\|} \frac{1}{N_{R} N_{P}} \sum_{i=1}^{N_{R}} \sum_{j=1}^{N_{P}} \frac{\int_{u=0}^{u=t} \sqrt{\sum_{k=2}^{d}\left[x_{k}^{j, i}(u)-x_{k}^{j, i}(u=0)\right]^{2}} \mathrm{~d} u}{t}$,

where we recall that $x_{k}^{j, i}(u)$ is the position of the particle $j$ in the simulation $i$ in the direction $k(k=2,3)$ at time $u$ and $d$ is the Euclidean dimension of the embedding space. In Figure $5, \tau(t)$ is the characteristic transverse scale of the cylinder in 3-D or of the tube in 2-D within which the flow line has expanded from the onset of the simulation to the time $t$. $\tau$ is much large in 3-D than in 2-D and it keeps increasing in 3-D while it converges quickly in 2-D. The convergence in 2-D is expected and is consistent with the vanishing transverse dispersion. Asymptotically, $\tau$ gives the typical width of the flow tube within which the flow lines remain confined. In 3-D, flow lines expand within a widening cone and their extension in the direction transverse to the main flow direction is not limited. By itself, this characteristic of the flow lines qualitatively explains the nonvanishing 3-D transverse dispersion. We underline that this transverse dispersion mechanism does not require any local diffusion or dispersion to be activated as it solely relies on the structure of the flow lines. Here it is activated by the ergodic nature of the injection conditions. It will be likely enhanced by any additional local diffusion or dispersion. It could also be critically be enhanced by temporal fluctuations of the flow conditions, which are already known to be an alternative cause of nonvanishing transverse dispersion in presence of heterogeneity [Rehfeldt and Gelhar, 1992].

[20] The longitudinal macrodispersivity is also critically impacted by the Euclidean dimension. It is much larger in 3-D than in 2-D for $\sigma_{Y}^{2} \geq 2.25$. However, it does not display the same type of behavior as the transverse macrodispersivity. It increases exponentially with $\sigma_{Y}^{2}$ (equation (9)) while the transverse macrodispersivity increases quadratically and it converges more slowly to the asymptotic regime. This latter observation has also been reported for 2-D heterogeneous systems submitted to temporal velocity fluctuations [de Dreuzy et al., 2012a]. Because of these significant differences in the behaviors, the mechanisms at the origin of the longitudinal and transverse macrodispersivities are not identical. The braiding of the flow lines responsible for the transverse dispersion may be either superseded or supplemented by other mechanisms like the long-range correlations of the velocity field promoted by heterogeneity [Le Borgne et al., 2007, 2008a].

[21] In fact, the Lagrangian correlation structure of the velocity field in the longitudinal direction has proven to have a strong influence on the longitudinal macrodispersion. If some of the correlation is destroyed by either diffusion, dispersion or temporal fluctuations, the longitudinal macrodispersion drops significantly [Beaudoin et al., 2010; de Dreuzy et al., 2012a]. As the dependence of the spatial correlation length of the velocity field to its magnitude can be linked to the upscaled dispersion using a correlated CTRW framework [Le Borgne et al., 2008a, 2008b], we plan to compare the 2-D and 3-D Lagrangian velocity correlations to explain the strong increase of the longitudinal dispersion from 2-D to 3-D. We finally expect significant impact of the dimensionality on mixing as mixing has already been found to be highly sensitive both to the longitudinal and transverse dispersions [Chiogna et al., 2011; de Dreuzy et al., 2012b; Rolle et al., 2009].

[22] Acknowledgments. J.-R.d.D. acknowledges the European Union for its additional funding through the IEF Marie-Curie fellowship (PIEFGA-2009-251710). Additional funding was provided by the French National Research Agency ANR through the H2MNO4 project (ANR- 
MN-2012). Numerical simulations benefited from the access to the HPC resources of IDRIS under the allocation 2012066203 made by GENCI (Grand Equipement National de Calcul Intensif).

\section{References}

Attinger, S., M. Dentz, and W. Kinzelbach (2004), Exact transverse macro dispersion coefficients for transport in heterogeneous porous media, Stoch. Environ. Res. Risk Assess., 18(1), 9-15.

Bear, J. (1973), Dynamics of Fluids in Porous Media, Dover Publications, Mineola, N. Y.

Beaudoin, A., J.-R. de Dreuzy, J. Erhel, and H. Mustapha (2005), Parallel simulations of underground flow in porous and fractured media, paper presented at Proceedings of the International Conference ParCo, John von Neumann Institute for Computing, Jülich.

Beaudoin, A., J.-R. de Dreuzy, and J. Erhel (2006), A comparison between a direct and a multigrid sparse linear solvers for highly heterogeneous flux computations, paper presented at European Conference on Computational Fluid Dynamics, ECCOMAS CFD 2006.

Beaudoin, A., J.-R. de Dreuzy, and J. Erhel (2007), An efficient parallel tracker for advection-diffusion simulations in heterogeneous porous media, Lecture Notes in Computer Science, 4641, 717-726

Beaudoin, A., J.-R. de Dreuzy, and J. Erhel (2010), Numerical Monte Carlo analysis of the influence of pore-scale dispersion on macrodispersion in 2-D heterogeneous porous media, Water Resour. Res., 46, W12537, doi:10.1029/2010WR009576.

Bellin, A., P. Salandin, and A. Rinaldo (1992), Simulation of dispersion in heterogeneous porous formations: Statistics, first-order theories, convergence of computations, Water Resour. Res., 28(9), 2211-2227.

Chavent, G., and J. E. Roberts (1991), A unified physical presentation of mixed, mixed-hybrid finite elements and standard finite difference approximations for the determination of velocities in waterflow problems, Adv. Water Resour., 14(6), 329-348.

Chin, D. A., and T. Wang (1992), An investigation of the validity of firstorder stochastic dispersion theories in isotropic porous media, Water Resour. Res., 28(6), 1531-1542.

Chiogna, G., O. A. Cirpka, P. Grathwohl, and M. Rolle (2011), Transverse mixing of conservative and reactive tracers in porous media: Quantification through the concepts of flux-related and critical dilution indices, Water Resour. Res., 47, W02505, doi:10.1029/2010WR009608.

Cirpka, O. A., and P. K. Kitanidis (2000), Characterization of mixing and dilution in heterogeneous aquifers by means of local temporal moments, Water Resour. Res., 36(5), 1221-1236.

Cirpka, O. A., F. P. J. de Barros, G. Chiogna, M. Rolle, and W. Nowak (2011), Stochastic flux-related analysis of transverse mixing in twodimensional heterogeneous porous media, Water Resour. Res., 47, W06515, doi:10.1029/2010WR010279.

Dagan, G. (1984), Solute transport in heterogeneous porous formations, $J$. Fluid Mech., 145, 151-177.

Dagan, G. (1988), Time-dependent macrodispersion for solute transport in anisotropic heterogeneous aquifers, Water Resour. Res., 24(9), 1491-1500.

Dagan, G. (1989), Flow and Transport in Porous Formations, 465 pp., Springer.

de Dreuzy, J.-R., A. Beaudoin, and J. Erhel (2007), Asymptotic dispersion in 2D heterogeneous porous media determined by parallel numerical simulations, Water Resour. Res., 43, W10439, doi:10.1029/2006WR005394.

de Dreuzy, J.-R., J. Carrera, M. Dentz, and T. Le Borgne (2012a), Asymptotic dispersion for 2D highly heterogeneous permeability fields under temporally fluctuating flow conditions, Water Resour. Res., 48, W01532, doi:10.1029/2011WR011129.

de Dreuzy, J.-R., J. Carrera, M. Dentz, and T. Le Borgne (2012b), Time evolution of mixing in heterogeneous porous media, Water Resour. Res., 48, W06511, doi:10.1029/2011WR011360.

de Simoni, M., J. Carrera, X. Sánchez-Vila, and A. Guadagnini (2005), A procedure for the solution of multicomponent reactive transport problems, Water Resour. Res., 41, W11410, doi:10.1029/2005WR004056.

Delhomme, J. P. (1979), Spatial variability and uncertainty in groundwaterflow parameters - Geostatistical approach, Water Resour. Res., 15(2), 269-280.

Dentz, M., H. Kinzelbach, S. Attinger, and W. Kinzelbach (2000), Temporal behavior of a solute cloud in a heterogeneous porous medium, 1, Point-like injection, Water Resour. Res., 36(12), 3591-3604.

Dentz, M., H. Kinzelbach, S. Attinger, and W. Kinzelbach (2002), Temporal behavior of a solute cloud in a heterogeneous porous medium: 3 . Numeri- cal simulations, Water Resour. Res., 38(7), 23-1-23-13, doi:10.1029/ 2001WR000436.

Englert, A., J. Vanderborght, and H. Vereecken (2006), Prediction of velocity statistics in three-dimensional multi-Gaussian hydraulic conductivity fields, Water Reosur. Res., 42, W03418, doi:10.1029/2005WR004014.

Erhel, J., J.-R. de Dreuzy, and E. Bresciani (2008), Multi-parametric intensive stochastic simulations for hydrogeology on a computational grid, in Parallel Computational Fluid Dynamics, Lecture Notes in Computational Science and Engineering, Springer.

Erhel, J., J.-R. de Dreuzy, A. Beaudoin, E. Bresciani, and D. Tromeur-Dervout (2009), A parallel scientific software for heterogeneous hydrogeoloy, paper presented at Parallel Computational Fluid Dynamics 2007, Lecture Notes in Computational Science and Engineering, edited by I.H. Tuncer, U. Gullcat, D. R. Emerson, and K. Matsuno, pp. 39-48, Springer-Verlag, Berlin.

Falgout, R. D., et al. (2005), Pursuing scalability for HYPRE's conceptual interfaces, ACM Trans. Math. Software, 31(3).

Freeze, R. A. (1975), Stochastic-conceptual analysis of one-dimensional groundwater flow in nonuniform homogeneous media, Water Resour. Res., 11(5), 725-741.

Frigo, M., and S. G. Johnson (2005), The design and implementation of FFTW3, paper presented at the IEEE, 93 (Special Issue on Program Generation, Optimization, and Platform Adaptation).

Gelhar, L. W. (1993), Stochastic Subsurface Hydrology, Engelwood Cliffs, N. J.

Gelhar, L. W., and C. L. Axness (1983), Three-dimensional stochastic analysis of macrodispersion in aquifers, Water Resour. Res., 19, 161-180.

Gutjahr, A. L. (1989), Fast fourier transforms for random field generation, Project Report for Los Alamos Grant to New Mexico Tech, Contract number 4-R58-2690R, Department of Mathematics, New Mexico Tech, Socorro, New Mexico.

Hsu, K. C. (2003), The influence of the log-conductivity autocovariance structure on macrodispersion coefficients, J. Contam. Hydrol., 65(1-2), 65-77.

Jankovic, I., A. Fiori, and G. Dagan (2003), Flow and transport in highly heterogeneous formations: 3. Numerical simulations and comparison with theoretical results, Water Resour. Res., 39(9), 1270, doi:10.1029/ 2002 WR001721.

Jankovic, I., D. R. Steward, R. J. Barnes, and G. Dagan (2009), Is transverse macrodispersivity in three-dimensional groundwater transport equal to zero? A counterexample, Water Resour. Res., 45, W08415, doi:10.1029/ 2009WR007741.

Jha, B., L. Cueto-Felgueroso, and R. Juanes (2011), Quantifying mixing in viscously unstable porous media flows, Phys. Rev. E, 84(6 Pt 2), 066312.

Kinzelbach, W. (1988), The random-walk method in pollutant transport simulation, in Groundwater Flow and Quality Modelling, NATO ASI Series $C, V .224$, edited by E. Custodia, A. Gurgui, and J. P. Lobo Ferreira, p. 227, Springer, New York.

Lallemand-Barres, A., and P. Peaudecerf (1978), Recherche des relations entre la valeur de la dispersivité macroscopique d'un milieu aquifère, ses autres caractéristiques et les conditions de mesure, Bull. Bur. Rech. Geol. Min., Sect 3, 4(4).

Le Borgne, T., J.-R. de Dreuzy, P. Davy, and O. Bour (2007), Characterization of the velocity field organization in heterogeneous media by conditional correlations, Water Resour. Res., 43, W02419, doi:10.1029/ 2006WR004875.

Le Borgne, T., et al. (2008a), Lagrangian statistical model for transport in highly heterogeneous velocity fields, Phys. Rev. Lett., 101(9), 4.

Le Borgne, T., et al. (2008b), Spatial Markov processes for modeling Lagrangian particle dynamics in heterogeneous porous media, Phys. Rev. E, $78(2), 9$.

Le Borgne, T., et al. (2010), Non-Fickian mixing: Temporal evolution of the scalar dissipation rate in heterogeneous porous media, Adv. Water Resour., 3(12), 1468-1475.

Le Borgne, T., et al. (2011), Persistence of incomplete mixing: A key to anomalous transport, Phys. Rev. E, 84(1), 015301.

Lunati, I., S. Attinger, and W. Kinzelbach (2002), Macrodispersivity for transport in arbitrary nonuniform flow fields: asymptotic and preasymptotic results, Water Resour. Res., 38(10), 1187, doi:10.1029/2001WR001203.

Matheron (1967), Eléments Pour une Théorie des milieux Poreux, Masson, Paris.

Neuman, S., C. L. Winter, and C. M. Newman (1987), Stochastic-theory of field-scale Fickian dispersion in anisotropic porous-media, Water Reosur. Res., 23(3), 453-466. 


\section{BEAUDOIN AND DE DREUZY : 3-D MACRODISPERSION}

Neuman, S. P., and Y.-K. Zhang (1990), A quasi-linear theory of non-fickian and fickian subsurface dispersion 1 . Theoretical analysis with application to isotropic media, Water Resour. Res., 26(5), 887-902.

Pollock, D. W. (1988), Semianalytical computation of path lines for finitedifference models, Ground Water, 26(6), 743-750.

Rehfeldt, K. R., and L. W. Gelhar (1992), Stochastic analysis of dispersion in unsteady flow in heterogeneous aquifers, Water Resour. Res., 28(8), 2085-2099.

Roberts, J.-E., and J.-M. Thomas (1991), Mixed and hybrid methods, in Handbook of Numerical Analysis 2, Finite Element Methods -part 1, edited by P. G. Ciarlet and J. L. Lions, pp. 523-639, Elsevier Sci.

Rolle, M., et al. (2009), Enhancement of dilution and transverse reactive mixing in porous media: Experiments and model-based interpretation, $J$. Contam. Hydrol., 110(3-4), 130-142.

Rubin, Y. (1990), Stochastic modeling of macrodispersion in heterogeneous porous media, Water Resour. Res., 26(1), 133-141.

Saffman, P. G. (1959), A theory of dispersion in a porous medium, J. Fluid Mech., 6, 321-349.

Salandin, P., and V. Fiorotto (1998), Solute transport in highly heterogeneous aquifers, Water Resour. Res., 34(5), 949-961.
Schwarze, H., et al. (2001), Estimation of macrodispersion by different approximation methods for flow and transport in randomly heterogeneous media, Transp. Porous Media, 43(2), 265-287.

Taylor, G. (1953), Dispersion of Soluble Matter in Solvent Flowing Slowly through a Tube, Proc. R. Soc. London Ser. A, 219(1137), 186-203.

Tompson, A. F. B., and L. W. Gelhar (1990), Numerical simulation of solute transport in three-dimensional, randomly heterogeneous porous media, Water Resour. Res., 26(10), 2541-2562.

Trefry, M. G., F. P. Ruan, and D. McLaughlin (2003), Numerical simulations of preasymptotic transport in heterogeneous porous media: Departures from the Gaussian limit, Water Resour. Res., 39(3), doi:10.1029/ 2001WR001101.

van Kampen, N. G. (1981), Stochastic Processes in Physics and Chemistry, Elsevier Sci.

Wen, X. H., and J. J. Gomez-Hernandez (1996), The constant displacement scheme for tracking particles in heterogeneous aquifers, Ground Water, 34(1), 135-142.

Zhang, Y.-K., and B.-M. Seo (2004), Numerical simulations of non-ergodic solute transport in three-dimensional heterogeneous porous media, Stoch. Environ. Res. Risk Assess, 18, 205-215. 